Arch. Tierz., Dummerstorf 42 (1999) 6, 527-533

Aus der Landwirtschaftskammer Hannover ${ }^{1)}$ und dem Institut fur Tierzucht und Tierverhalten Mariensee ${ }^{2)}$

WILFRIED BRADE") und EILDERT GROENEVELD ${ }^{2)}$

\title{
Wechselwirkung zwischen Vater und Mutter in der Milchrinderzüchtung
}

\author{
Herrn Professor Dr. Peter Glodek zum 65. Geburtstag gewidmet
}

\begin{abstract}
Summary
Title of the paper: Interaction between sire and dam in dairy cattle breeding

The traditional animal model in genetic evaluation has been extended by a sire * dam interaction component demonstrating the computational feasibility.

The expected additional information response from including a sire * dam component is small, however, this effect come larger with an increase in the number of animals produced by biotechnical means which will likely get special treatments.
\end{abstract}

Key Words: dairy yield, heritabilities, genetic evaluation, REML, sire * dam interaction, BLUP

\section{Zusammenfassung}

In der vorliegenden Arbeit wurde das üblicherweise verwendete Tiermodell zur Ermittlung von Zuchtwerten um die Vater * Mutter-Interaktionskomponente erweitert. Die rechentechnische Bewältigung eines derartigen Datenmaterial konnte demonstriert werden. Der dadurch erzielte zusätzliche Informationsgewinn blieb gering, könnte jedoch vor dem Hintergrund künftiger Entwicklungen in der Rinderzucht (Zunahme biotechnischer Maßnahmen, mögliche Sonderbehandlungen von Tiergruppen, Familien) - bei Auswertung von Feldmaterial an Bedeutung gewinnen.

Schlïsselwörter: Milchertrag, Heritabilität, Zuchtwertschätzung, REML, Vater * Mutter-Interaktion, BLUP

\section{Einführung}

In der aktuellen Zuchtwertschätzung bei Milchrindern wird davon ausgegangen, daß die gesamte genetische Varianz auf additiven Genwirkungen beruht. Das regelmäßige Auftreten beispielsweise von Inzuchtdepressionen - nach Verpaarung von verwandten Tieren - ist aber ein Beleg, daß nichtadditive Genwirkungen wirksam sind.

Die konsequente Nutzung moderner Biotechniken (Embryotransfer, Clonierung etc.) führen gleichzeitig zur Zunahme engverwandter Tiere, vor allem in der höchsten Zuchtstufe. Nachfolgend soll eine einfache Vorgehensweise zur zusätzlichen Erfassung der nichtadditiv genetischen Variabilität für verschiedene Milchleistungsmerkmale aufgrund der Interaktionskomponente Vater * Mutter an einem Datenmaterial des Osnabrücker Herdbuchs (OHG) aufgezeigt werden. 
BRADE; GROENEVELD: Wechselwirkung zwischen Vater und Mutter in der Milchrinderzüchtung

2. Material und Methodik

Das Datenmaterial basiert auf 18772 erste Laktationsleistungen von Holstein-Kühen des Osnabrücker Zuchtgebietes (OHG) aus dem Abkalbezeitraum 1993/94 mit 79530 Tieren im Pedigree-File.

Eine Übersicht zum Datenmaterial enthält Tabelle 1:

Tabelle 1

Ausgewertetes Datenmaterial (1.Laktation) - phänotypische Mittelwerte und Standardabweichungen (s)

(Used data-1st lactation, phenotypic means and standard deviations )

\begin{tabular}{lcc}
\hline \multicolumn{1}{c}{ Merkmal (Abkürzung) } & phänotypisches Mittel & Standardabweichung (s) \\
\hline Milchmenge (M-kg) & $6.785,7$ & $1.396,0$ \\
Eiweißmenge (P-kg) & 222,7 & 45,5 \\
Eiweißgehalt (P-\%) & 3,29 & 0,20 \\
& & \\
\hline Anzahl Tiere mit Leistung: & 18.772 & \\
Anzahl Tiere im Pedigree-File: & 79.530 & \\
Anzahl Herden: & 1.530 & \\
\hline
\end{tabular}

Eine erste Auswertung der Daten erfolgte zunächst nach einem 3-Merkmals-Tiermodell:

$P(M-k g)_{i j k}=h_{i}+s_{j}+a_{k}+b_{E K A}\left(E K A_{i j k}-E K A\right)+b_{305}\left(t_{i j k}-t\right)+e_{i j k}$

$P(P-k g)_{i j k}=h_{i}+s_{j}+a_{k}+b_{E K A}\left(E K A_{i j k}-E K A\right)+b_{305}\left(t_{i j k}-t\right)+e_{i j k}$

$P(P-\%)_{i j k}=h_{i}+s_{j}+a_{k}+b_{E K A}\left(E K A_{i j k}-E K A\right)+b_{305}\left(t_{i j k}-t\right)+e_{i j k}$

wenn

$\mathrm{P}(\mathrm{M}-\mathrm{kg})_{\mathrm{ijk}}=$ Merkmalswert für die Milchmenge einer auszuwertenden Kuh (analog: P (P-kg) $)_{i j k}$ für die Eiweißmenge bzw. P (P-\%) $)_{i j k}$ für den Eiweißgehalt)

$\mathrm{h}_{\mathrm{i}} \quad=\mathrm{i}$-te Herde (fixer Effekt)

$\mathrm{s}_{\mathrm{j}} \quad=\mathrm{j}$-ter Kalbesaison-Effekt (fixer Effekt)

$a_{k}=$ zufälliger Effekt des k-ten Tieres (unter Einbeziehung der additivgenetischen Verwandtschaftsmatrix)

$\begin{aligned} \mathrm{EKA}_{\mathrm{ijk}}= & \begin{array}{l}\text { Erstkalbealter (Kovariable) für das betreffende Tier }(23 \leq \mathrm{EKA} \leq 38 \mathrm{Mo}- \\ \text { nate) }\end{array}\end{aligned}$

$\mathrm{t}_{\mathrm{ijk}}=$ Anzahl Melktage in der ersten 305-Tage-Laktation, definiert als Kovariable $(240 \leq \mathrm{t} \leq 305$ Tage $)$ Das obige Gleichungssystem kann - unter Verwendung der Matrixnotation- auch wie folgt verallgemeinert
dargestellt werden:

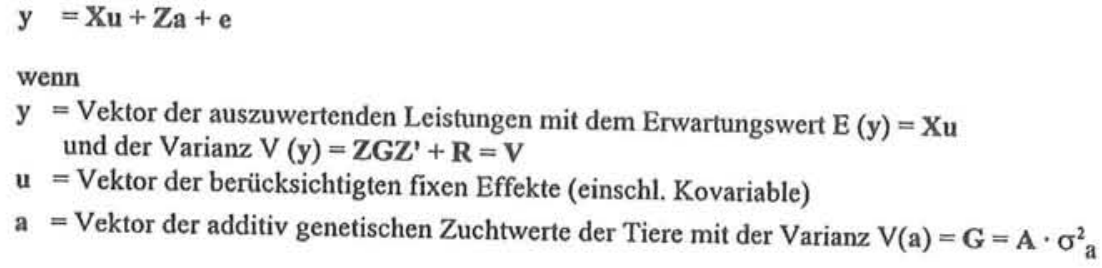




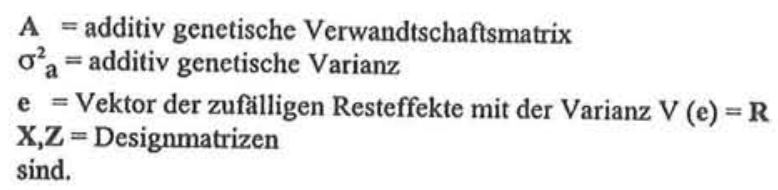

In einer zweiten Auswertung des Datenmaterials erfolgte eine Erweiterung dieses Modells um die Interaktionskomponente Vater * Mutter, die gleichfalls zufällig definiert wurde und vorrangig der Erfassung nichtadditiver Effekte diente (,,erweitertes Tiermodell").

Gleichzeitig erfolgte die Einbeziehung des Inzuchtniveaus (F) auszuwertender Tiere: $P(M-k g)_{i j k l}=h_{i}+s_{j}+a_{k}+f \bullet m_{1}+b_{E K A}\left(E K A_{i j k l}-E K A\right)+b_{30 s}\left(t_{i j k l}-t\right)+b_{i n z .}\left(F_{i j k l}-F\right)+e_{i j k l}$ $P(P-k g)_{i j k l}=h_{i}+s_{j}+a_{k}+f \circ m_{1}+b_{E K \Lambda}\left(E K A_{i j k l}-E K A\right)+b_{305}\left(t_{i j k l}-t\right)+b_{i n z .}\left(F_{i j k l}-F\right)+e_{i j k l}$ $P(P-\%)_{i j k l}=h_{i}+s_{j}+a_{k}+f \bullet m_{1}+b_{E K A}\left(E K A_{i j k 1}-E K A\right)+b_{305}\left(t_{i j k l}-t\right)+b_{i n z .}\left(F_{i j k l}-F\right)+e_{i j k l}$
wenn jetzt

$\mathrm{f} \bullet \mathrm{m}_{1}=$ Interaktionskomponente Vater mal Mutter (zufälliger Effekt) mit dem Erist. wartungswert $E(f \circ m)=0$ und der Varianz $V(f \circ m)=\sigma_{f \cdot m}^{2}$

Die Interpretation der Vater * Mutter-Interaktionskomponente ist schwierig:

Die Vater * Mutter-Komponente erfaßt die zusätzliche Vollgeschwisterähnlichkeit aufgrund nichtadditiver Wirkungen (identische Mehrlinge infolge von Splitting von Embryonen bzw. Clonierung ausgeschlossen), die bei Vollgeschwistern mit einem Viertel der vorhandenen Dominanzvarianz angegeben werden kann (FALCONER, 1984). Darüber hinaus sind weitere epistatische Komponenten zusätzlich an der Vollgeschwisterähnlichkeit beteiligt, die nicht klar von der Dominanzkomponente zu trennen sind (FALCONER, 1984).

Eine korrekte genetische Interpretation dieser Komponente wird aber vor allem dadurch erschwert, daß Tiere aus einer gezielten Anpaarung einschl. Embryotransfer (ET) häufig eine zusätzliche (= „bewußte") Sonderbehandlung in den Züchterställen erfahren (= kostenintensive Reproduktionsverfahren), so daß eine spezifische (permanente) Umweltkomponente $\left(\mathrm{s}^{2}\right.$ spez. Umwelt $)$ diese Interaktionskomponente „aufblähen“ kann.

In der Literatur neuerdings zu findende Schätzwerte bzgl. der Höhe der Dominanzvarianz für Milchleistungsmerkmale berücksichtigen diese Tatsache häufig nicht genügend.

Die zusätzliche Beachtung der Vater * Mutter-Interaktion könnte somit gleichzeitig eine Möglichkeit sein, derartige „Sonderbehandlungen“ zumindest ansatzweise zu berücksichtigen.

Die erforderlichen genetischen Populationsparameter wurden mit dem Programmpaket VCE 3.2 geschätzt, das eine Implementierung des multivarianten REML darstellt (GROENEVELD, 1996). Interessierende Zuchtwerte wurden anschließend mit dem Programmpaket PEST ermittelt (GROENEVELD, 1995). Zu erwähnen bleibt, daß die notwendigen Rechenarbeiten auf einer SUN 670 mit $50 \mathrm{MHz}$ getaktetem Super Sparc Prozessoren im Institut Mariensee erfolgten. 
3. Ergebnisse

3.1 Geschätzte Varianzkomponenten

Die geschätzten Varianzkomponenten - ohne bzw. mit Einbeziehung der Vater * Mutter-Interaktion $\left(\mathrm{s}^{2}{ }_{\mathrm{f}} \cdot \mathrm{m}\right)$ - enthält Tabelle 2 .

Tabelle 2

Ergebnisse der multivariaten Varianzanalysen einschl. zugehörige Standardfehler (Multivariate analysis of data and standard errors)

\begin{tabular}{|c|c|c|c|c|c|c|}
\hline \multirow[t]{2}{*}{ Parameter } & \multicolumn{2}{|c|}{ M-kg } & \multicolumn{2}{|c|}{ P-kg } & \multicolumn{2}{|c|}{$\mathrm{P}-\%$} \\
\hline & Modell 1 & $\begin{array}{l}\text { Modell } 2 \\
\text { (erweitert) }\end{array}$ & Modell 1 & $\begin{array}{l}\text { Modell } 2 \\
\text { (erweitert) }\end{array}$ & Modell 1 & $\begin{array}{l}\text { Modell } 2 \\
\text { (erweitert) }\end{array}$ \\
\hline $\mathrm{s}_{\text {rexidual }}^{2}$ & $\begin{array}{l}516.690,97 \\
(19.434,74)\end{array}$ & $\begin{array}{l}464.482,67 \\
(25.941,35)\end{array}$ & $\begin{array}{l}491,07 \\
(16,33)\end{array}$ & $\begin{array}{l}443,62 \\
(20,90)\end{array}$ & $\begin{array}{c}0,0105 \\
(0,0008)\end{array}$ & $\begin{array}{c}0,0088 \\
(0,0011)\end{array}$ \\
\hline $\mathrm{s}^{2}{ }_{\text {tier }}$ & $\begin{array}{l}436.296,23 \\
(27.613,53)\end{array}$ & $\begin{array}{l}434.664,65 \\
(26.539,08)\end{array}$ & $\begin{array}{l}333, .60 \\
(22,95)\end{array}$ & $\begin{array}{l}329,09 \\
(20,25)\end{array}$ & $\begin{array}{c}0,0243 \\
(0,0012)\end{array}$ & $\begin{array}{c}0,0238 \\
(0,0012)\end{array}$ \\
\hline $\mathrm{s}^{2} \mathrm{f}^{\bullet} \mathrm{m}$ & - & $\begin{array}{c}51.719,96 \\
(22.178,61)\end{array}$ & - & $\begin{array}{c}49,24 \\
(18,20)\end{array}$ & - & $\begin{array}{c}0,0020 \\
(0,0008)\end{array}$ \\
\hline $\mathrm{s}^{2}{ }_{\text {tier }} / \mathrm{s}^{2}$ gessumt & $\begin{array}{c}0,458 \\
(0,024)\end{array}$ & $\begin{array}{c}0,457 \\
(0,024)\end{array}$ & $\begin{array}{c}0,405 \\
(0,0024)\end{array}$ & $\begin{array}{c}0,400 \\
(0,022)\end{array}$ & $\begin{array}{c}0,697 \\
(0,028)\end{array}$ & $\begin{array}{c}0,690 \\
(0,029)\end{array}$ \\
\hline $\mathrm{s}^{2}{ }_{\mathrm{f}}{ }^{\mathrm{m}} / \mathrm{s}_{\mathrm{gesam}}{ }$ & - & $\begin{array}{c}0,054 \\
(0,023)\end{array}$ & - & $\begin{array}{c}0,060 \\
(0,023)\end{array}$ & - & $\begin{array}{c}0,057 \\
(0,023)\end{array}$ \\
\hline
\end{tabular}

Die in der Tabelle 2 zusammengefaßten Werte belegen, daß die Wechselwirkungskomponente $\left(\mathrm{s}^{2}{ }_{\mathrm{f}} \cdot \mathrm{m}\right)$ vom Rest $\left(\mathrm{s}^{2}\right.$ residual $)$ und nicht von der Tiervarianz $\left(\mathrm{s}^{2}{ }_{\text {tier }}\right)$ „abgespalten“ wurde.

In jedem Fall wurde eine spürbare Reduzierung der Restvarianz $\left(\mathrm{s}^{2}{ }_{\text {residual }}\right)$ erreicht.

Der Varianzanteil für die Interaktion Vater * Mutter $\left(\mathrm{s}^{2}{ }_{\mathrm{f}}{ }^{\circ} \mathrm{m} / \mathrm{s}^{2}\right.$ gesamt $)$ beträgt im vorliegenden Datenmaterial ca. 5- $6 \%$ (Tab. 2).

Gleichzeitig sind die Heritabilitäten $\left(\mathrm{h}^{2}{ }_{\text {tier }}=\mathrm{s}^{2}{ }_{\text {tier }} / \mathrm{s}^{2}\right.$ gesamt $)$ im einfachen Tiermodell (Modell 1) nur unbedeutend höher als im „erweiterten Tiermodell“ (Modell 2).

Die im erweiterten Tiermodell (Modell 2) zusätzlich ermittelten Inzuchtdepressionen (anhand der 18772 Tiere mit einer Eigenleistung) enthält Tabelle 3.

Tabelle 3

Inzuchtdepressionen (lineare Regression) (Inbreeding depression (lineare regression))

\begin{tabular}{lc}
\hline Merkmal & b (je 1\% Inzuchtzunahme) \\
\hline Milchmenge (M-kg) & $-27.285 \mathrm{~kg}$ \\
Eiweißmenge (P-kg) & $-0,783 \mathrm{~kg}$ \\
Eiweißgehalt (P-\%) & $+0,0004 \%$
\end{tabular}

Je 1\%-Inzuchtzunahme ist mit einer Abnahme der Milch- bzw. -eiweißmenge von 27,3 $\mathrm{kg}$ Milch bzw. 0,78 kg Eiweiß zu rechnen.

Ähnliche Werte haben kürzlich WIGGANS u.a. (1995) für die US-Holstein-Population mitgeteilt.

Die ermittelten genetischen Korrelationen $\left(r_{g}\right)$ - nach den verschiedenen Modellansät- 
Tabelle 4

Genetische Merkmalsbeziehungen in verschiedenen Modellen $\left(\mathrm{r}_{\mathrm{g}}\right)$ (Genetic correlations in different models $\left.\left(\mathrm{r}_{\mathrm{g}}\right)\right)$

\begin{tabular}{lccc}
\hline & M-kg : P-kg & M-kg: P-\% & P-kg: P-\% \\
\hline Modell 1 (Tiermodell) & & & $-0,088$ \\
$\mathrm{r}_{\mathbf{B} \text { (tier) }}$ & 0,875 & $-0,558$ & $(0,042)$ \\
& $(0,008)$ & $(0,029)$ & \\
Modell 2 (erweitertes Tiermodell) & & & $-0,093$ \\
$\mathrm{r}_{\mathbf{8} \text { (tier) }}$ & 0,883 & $-0,550$ & $(0,042)$ \\
\hline
\end{tabular}

zen - enthält Tabelle 4.

$\mathrm{Zu}$ vermerken ist, daß die genetischen Merkmalsbeziehungen aufgrund der Tierkomponente $\left(\mathrm{r}_{\mathrm{g} \text {-tier }}\right)$ in den verschiedenen Modellansätzen wenig differieren.

Die genetischen Zusammenhänge zwischen Milch- und Milcheiweißgehalt sind, wie zu erwarten war, eindeutig negativ. Interessanterweise sind aber gleichzeitig auch negative Beziehungen zwischen Milcheiweißmenge und -gehalt zu nennen.

\subsection{Simulierte Selektion}

Schließlich wurden die Selektionsdifferenzen (SD) bestimmt, wenn jeweils ein konstanter Kuhanteil nach der Höhe der Zuchtwerte (BLUP-Zuchtwerte) ausgelesen wird (Tab. 5).

Tabelle 5

Realisierte Selektionsdifferenzen (Realised selection differences)

\begin{tabular}{|c|c|c|c|}
\hline \multirow[t]{2}{*}{ Ausleseanteil } & \multirow[t]{2}{*}{ Merkmal } & \multicolumn{2}{|c|}{ realisierte Selektionsdifferenzen (SD) } \\
\hline & & $\begin{array}{l}\text { Tiermodell } \\
\text { (Modell 1) }\end{array}$ & $\begin{array}{l}\text { erw. Tiermodell } \\
\text { (Modell 2) }\end{array}$ \\
\hline $1.877 / 18.772$ & Milch-kg & $+912,92$ & $\begin{array}{c}+915,10 \\
(=+0,24 \%)\end{array}$ \\
\hline $1.877 / 18.772$ & Eiweiß-kg & $+23,57$ & $\begin{array}{c}+23,75 \\
(=+0,76 \%)\end{array}$ \\
\hline $1.877 / 18.772$ & Eiweiß-\% & $+0,158$ & $\begin{array}{c}+0,158 \\
(= \pm 0,00 \%)\end{array}$ \\
\hline
\end{tabular}

Der zusätzliche Selektionsgewinn beträgt für die Mengenmerkmale 0,2 - 0,8\% (Tab. 5). Er ist zweifellos in der absoluten Größenordnung nur gering, bestätigt jedoch damit die prinzipielle Vorzüglichkeit des erweiterten Tiermodells.

Berücksichtigt man, daß eine weitere Zunahme biotechnischer Maßnahmen in der höchsten Zuchtstufe beim Rind zu erwarten ist und Sonderbehandlungen für derartig erzeugte Zuchttiere - bei Feldprüfungen im Züchterstall - nicht ausgeschlossen werden können, bleiben derartig erweiterte Modellansätze ein mögliches Mittel der Wahl.

4.

Diskussion

Die in der vorliegenden Arbeit - auf der Basis multivariater Tiermodelle unter Ver- 
wendung von REML als Schätzverfahren - berechneten Heritabilitäten sind für die untersuchten Mengenmerkmale mit $h^{2}$ tier $\geq 0,4$ und für den Milcheiweißgehalt mit $h^{2}$ tier $\geq 0,6$ anzugeben.

Diese Werte sind damit deutlich größer als frühere Auswertungen im OHG-Zuchtbereich beispielsweise aufgrund einfacher väterlicher Halbgeschwisteranalysen (KANDZI, 1989, u.a.). Die Ursachen für diese Unterschiede sind durch die differenzierten Modellansätze und Schätzverfahren zu erklären.

So ist bekannt, daß beispielsweise verschiedene Schätzverfahren mögliche Vorselektionseffekte unterschiedlich zu erfassen vermögen (vgl. z.B. VAN RADEN u.a., 1988).

Die in der vorliegenden Arbeit erfaßte Anteil für die Interaktionskomponente Vater * Mutter $\left(\mathrm{s}^{2}{ }_{\mathrm{f}} \cdot \mathrm{m} / \mathrm{s}^{2}\right.$ gesamt $)$ erreicht Werte von ca. 5-6\%.

Eine zusätzliche Einbeziehung dieser Interaktionskomponente läßt zugehörige Restvarianzen deutlich reduzieren.

Erhebliche Schwierigkeiten bereitet die korrekte Interpretation dieser Varianzkomponente.

Die Autoren nehmen an, daß durch mögliche Sonderbehandlungen, speziell eng verwandter Tiere aus ET (ET-Vollgeschwister), diese Varianzkomponente systematisch beeinflußt sein kann. Auf die Angabe der Höhe der nichtadditiven Varianz (Dominanzvarianz) für die untersuchten Merkmale wird deshalb hier bewußt verzichtet.

In der neueren Literatur verstärkt zu findende, z.T. überraschend hohe Schätzwerte für die Dominanzvarianz (TEMPELMAN u.a., 1990; FÜRST und SÖLKNER, 1994, u.a.m.) könnten hier ihre mögliche Ursache haben.

Die zusätzliche Berücksichtigung der Interaktionskomponente Vater * Mutter in der Zuchtwertschätzung und einer darauf aufbauenden Selektion läßt eine prinzipielle Zunahme zugehöriger Selektionsdifferenzen erwarten. Der dadurch mögliche zusätzliche Selektionsgewinn ist jedoch gering, könnte aber vor dem Hintergrund künftiger Entwicklungen in der Rinderzucht (Zunahme biotechnischer Maßnahmen) an Bedeutung gewinnen.

\section{Literatur}

FALCONER, D.S.:

Einführung in die quantitative Genetik. Verlag Eugen Ulmer, Stuttgart, 1984

FÜRST, CH.; SÖLKNER, J.:

Additive and nonadditive genetic variances for milk yield, fertility and lifetime performance traits in dairy cattle. J. Dairy Sci. 77 (1994), 114-1128

GROENEVELD, E.:

REML VCE - a Multivariate Model Restricted Maximum Likelihood (Co) Variance Component GROENEVELD, E.:

KANDZI, A.:

PEST User's Manual, Mariensee, 1995

Die Bedeutung der Einsatzleitung in der 1. Laktation für die Effizienz der Bullenmütter - Selektion im Rahmen neuer Rinderzuchtprogramme. Univ. Göttingen, Diss., 1989 
MIGLIOR, F.; BURNSIDE, E.B.; KENNEDY, B.W.:

Production traits of Holstein cattle: estimation of nonadditive genetic variance components and in breeding depression. J. Dairy Sci. 78 (1995), 1174-1180

\section{TEEPKER, G.:}

Untersuchungen über genetische Parameter und Gesamtindices für Milchleistungsmerkmale. Univ. Göttingen, Diss., 1988

TEMPELMAN, R.J.; BURNSIDE, E.B.

Additive and nonadditive genetic variation for production traits in Canadian Holsteins. J. Dairy Sci. 73 (1990), 2206-2215

VAN RADEN, P.M.; JUNG, Y.C.:

A general purpose approximation to restricted maximum likelihood: the tilde - hat - approach. J. Dairy Sci., (1988), 187-199

WIGGANS, G.R.; VAN RADEN, P.M.; ZUURBIER, J.:

Calculation and use of inbreeding coefficients for genetic evoluation of United States dairy cattle. J. Dairy Sci. 78 (1995), 1589-1590

Eingegangen: 26.02.1999

Akzeptiert: 28.09 .1999

Anschriften der Verfasser

Priv.-Doz. Dr. habil. WILFRIED BRADE

Landwirtschaftskammer Hannover

Johannssenstr. 10

D- 30159 Hannover

Dir. u. Prof. Dr. EILDERT GROENEVELD

Institut für Tierzucht $\mathrm{u}$. - verhalten

Höltystr. 10

D- 31535 Neustadt/Mariensee 
Arch. Tierz., Dummerstorf 42 (1999) 6, 534

Buchbesprechung

Pharmakotherapie bei Haus- und Nutztieren

WOLFGANG LÖSCHER, FRITZ RUPERT UNGEMACH und REINHARD KROCKER

4. durchgesehene Auflage, 485 Seiten, 13 Abbildungen, 67 Tabellen, Parey Buchverlag Berlin, 1999, ISBN 3 8263-3286-5, 128,00 DM, 715,00 öS; 90,50 sFr

Neun Jahre nach Ersterscheinen liegt nunmehr die 4. Auflage dieses, für den deutschen Sprachraum einmaligen, Standardwerkes der veterinärmedizinischen Pharmakotherapie vor. Es enthält Aktualisierungen, die sich aus den Veränderungen des Arzneimittelmarktes sowie von arzneimittelrechtlichen Bestimmungen ergaben. Ziel dieses bewährten Buches ist es Klinikern, Praktikern und Studierenden, neben pharmakologischen Grundlagen, Informationen über Warenzeichen, Dosierungen Tierartenunterschiede in den Wirkungen und der Wirkungsdauer von Arzneimittelgruppen für die Anwendung beim Tier übersichtlich zu vermitteln. In diesem Nachschlagewerk sind alle für die veterinärmedizinische Praxis wichtigen Arzneimittel in übersichtlichen Monographien dargestellt. Um die erwünschten oder unerwünschten Wirkungen einzelner Pharmaka zu erfassen, werden den einzelnen Monographien Erläuterungen vorangestellt, in denen in knapper Form pharmakologische Grundlagen und Wirkungsprinzipien sowie allgemeine Hinweise zu den einzelnen Wirkstoffgruppen enthalten sind. Bei den Arzneimitteln, die für die Anwendung beim Tier zugelassen sind, werden in erster Linie Monopräparate aufgeführt, während Kombinationspräparate nur dann genannt werden, wenn die Wirkstoffkombination aus pharmakologischer und therapeutischer Sicht sinnvoll erschien. Eine Reihe humanmedizinischer Fertigarzneimittel, die für die Anwendung beim Tier von Interesse sind, fanden ebenfalls Berücksichtigung.

Die einzelnen, sich auf notwendige Texte beschränkenden, Kurzmonographien tragen als Überschrift den Freinamen des jeweiligen Pharmakons eventuell ergänzt durch ein oder mehrere Handelsnamen von gängigen Präparaten des jeweiligen Pharmakons. Beschrieben werden u.a. Anwendungsgebiete, Dosierung bei den einzelnen Applikationsarten, Wirkungsdauer, Nebenwirkungen, Gegenanzeigen und Wechselwirkungen, notwendige Wartezeiten. Die Einteilung der Wirkstoffgruppen erfolgt nach pharmazeutischen Gesichtspunkten und ein im Anhang befindlicher sorgfältig ausgearbeiteter Index erleichtert wesentlich die Arbeit mit diesem Buch. Nach einleitenden Abschnitten zu Grundbegriffen der Pharmakologie und den arzneimittelrechtlichen Bestimmungen, folgt der umfangreiche, in 26 Hauptabschnitte gegliederte, Teil der speziellen Pharmakologie und -Pharmakotherapie. Darunter z.B. die Beschreibung von Pharmaka mit Wirkung auf das Nervensystem, Lokalanästhetika, herz-, kreislauf-, nierenwirksame Pharmaka, Pharmaka zur Beeinflussung der Uterusfunktion, des Respirationstrakts, der Leber, des Magen-Darmtraktes, Desinfektionsmittel, Pharmaka zur Behandlung und Verhütung bakterieller Infektionen, Antiparasitika, Vitamine und Spurenelemente, Pharmaka zur Beeinflussung von Entzündungen, zur Therapie wichtiger Vergiftungen, Antiprotozoika, Hömoopathika und weitere Gruppen. Ein Anhang enthält u.a. Hinweise zur Umrechnung von Humandosierungen für Tiere, zur Arzneimittelkombination, zur Arzneimittelanwendung bei Fischen, Geflügel sowie Labor- und Heimtieren.

Dieses informative, gut gegliederte und durch drucktechnische Hervorhebungen von wichtigen Begriffen besonders übersichtliche Standardwerk der veterinärmedizinischen Pharmakotherapie ist als unentbehrliches Fachbuch für jeden Tiermediziner, Kliniken und Behörden uneingeschränkt zu empfehlen.

ERNST RITTER; Dummerstorf 